\title{
Por Que Precisamos de um Novo Registro Nacional em Intervenções Coronárias Percutâneas?
}

\author{
Áurea J. Chaves
}

$\mathbf{R}$ egistros são coleções prospectivas de dados, que, na cardiologia intervencionista, têm a finalidade de estudar a segurança e a eficácia das intervenções cardíacas percutâneas. Necessitam, como requisitos básicos, incluir pacientes consecutivos e obter dados completos e fidedignos, que caracterizem o paciente, o procedimento e os resultados clínicos, a curto e a longo prazos. Registros nacionais devem, adicionalmente, ser multicêntricos, abrangendo instituições públicas e privadas, e controlados por sociedades médicas que coordenem de forma independente a avaliação de seus resultados. Seus dados completam aqueles obtidos pelos estudos randomizados, avaliando se os resultados são reprodutíveis em larga escala, no amplo espectro de perfis clínicos e angiográficos da prática clínica. A Central Nacional de Intervenções Cardiovasculares (CENIC), órgão oficial da Sociedade Brasileira de Hemodinâmica e Cardiologia Intervencionista ( $\mathrm{SBHCl}$ ), criada em 1991, foi a primeira iniciativa nesses moldes e motivo de publicações que trouxeram os primeiros números representativos da experiência nacional com a intervenção coronária percutânea. Apesar da inquestionável contribuição à comunidade intervencionista brasileira, seus resultados são influenciados por um viés de seleção, em decorrência da natureza voluntária da inclusão de dados, e não conseguem, portanto, reproduzir perfeitamente a realidade dos números nacionais.

Novos projetos são necessários, e o artigo original do grupo encabeçado por Pedro Lemos, do Instituto do Coração do Hospital das Clínicas da Faculdade de Medicina da Universidade de São Paulo (São Paulo, SP), apresenta um novo sistema informatizado, acessível via web, desenvolvido para integrar uma rede interinstitucional destinada a coletar de forma detalhada dados de pacientes tratados no Brasil. O projeto conta com o apoio dos Ministérios da Ciência e Tecnologia e da Saúde, fundamental para garantir sua viabilidade. Oliveira e Avezum, do Instituto Dante Pazzanese de Cardiologia (São Paulo, SP), em editorial correspondente, apontam quais são os requisitos necessários para que um registro possa, de fato, mensurar a prática clínica nacional. O desafio seguinte estará em obter amostragem adequada dos centros participantes, de modo a refletir a realidade brasileira, e, depois, conseguir o acompanhamento a longo prazo dos pacientes incluídos.
Em outra excelente contribuição, Falcão et al., do Hospital de Clínicas Niterói (Niterói, RJ) e Hospital PróCardíaco (Rio de Janeiro, RJ), exploram a eficácia da trombólise intra-arterial e terapia endovascular adjunta em restabelecer o fluxo do vaso tratado na fase aguda do acidente vascular cerebral isquêmico, em pacientes selecionados. Trata-se de um grupo de hospitais com capacitação para o atendimento desses pacientes, onde a avaliação clínica é realizada por médico apto a classificar o paciente de acordo com a National Institute of Health Stroke Scale (NIHSS), as imagens são obtidas em tempo hábil (tomografia computadorizada em todos os casos e ressonância magnética de difusão, quando necessária) e a equipe de neurointervenção fica disponível para atuar dentro da janela de tempo preconizada. Bersin, do Seattle Cardiology and Swedish Medical Center (Seattle, Estados Unidos), em seu editorial, faz uma revisão das principais publicações que fundamentam a reperfusão no acidente vascular cerebral isquêmico, lembra que as diretrizes da American Heart Association para o tratamento do acidente vascular cerebral isquêmico ainda não recomendam o tratamento endovascular nesse cenário, e informa que os estudos IMS III, nos Estados Unidos, e COMBATSTROKE, na Europa, estão em andamento para comparar rt-PA intravenoso vs. rt-PA intra-arterial/intervenção mecânica no acidente vascular cerebral isquêmico.

Artigo de muito interesse, de Cardoso et al., do Instituto de Cardiologia do Rio Grande do Sul (Porto Alegre, RS), investiga o perfil e explora os resultados clínicos obtidos de uma coorte consecutiva de pacientes submetidos a intervenção coronária percutânea primária, tratados dentro e fora do horário regulamentar de trabalho de sua instituição, centro cardiológico de referência que disponibiliza o tratamento 24 horas por dia, sete dias por semana. Mattos, do Instituto Dante Pazzanese de Cardiologia, em editorial relacionado, comenta a influência de uma das principais variáveis envolvidas nos resultados da intervenção coronária percutânea primária, o tempo porta-balão, e de outra variável, menos explorada mas não menos importante, a experiência dos cardiologistas intervencionistas que atuam em plantões, feriados e finais de semana. Recomenda, ao final, uma série de medidas que as instituições devem adotar, para que se obtenham os melhores resultados no tratamento dessa condição, principal causa de mortalidade no Brasil. 
Outra área explorada nesta edição, muito original, diz respeito à utilização de plugs vasculares para a oclusão de vasos anômalos, tanto congênitos (colaterais, fístulas coronárias e pulmonares) como pós-cirúrgicos (anastomose de Blalock-Taussig). Pereira et al., do Instituto Dante Pazzanese de Cardiologia, trazem sua experiência e demonstram a versatilidade do tratamento endovascular na abordagem dessas estruturas vasculares diversas. Algumas das imagens do artigo, pelo seu ineditismo, foram selecionadas para ilustrar a capa da presente edição. Peirone, do Hospital Infantil de Córdoba (Córdoba, Argentina), em seu editorial, chama a atenção para a carência de publicações, na literatura, explorando esse tema, assim como para o cuidado na seleção dos casos tratados, o sucesso da técnica, mesmo em lesões com tortuosidades excessivas, e as peculiaridades das diversas gerações de plugs vasculares.
Finalizando, gostaria de chamar sua atenção para a excelência dos demais artigos originais publicados, que exploram temas muito atuais como o impacto do sangramento associado à intervenção coronária percutânea, o tratamento de coronárias de pequeno calibre, a utilização de cateteres dedicados ao acesso radial, as consequências do fenômeno de no-reflow, os resultados de um stent dedicado ao tratamento de enxertos de safena, o benefício do atendimento psicológico hospitalar pré-intervenção, o controle da emissão de radiação na sala de cateterismo, e a acurácia do cálculo da área valvar mitral pela ecocardiografia tridimensional.

Aproveitem!

Áurea J. Chaves Editora 